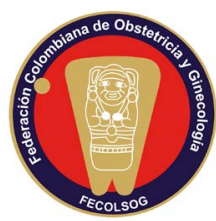

\title{
La importancia de la nutrición en la mujer gestante
}

\author{
Sara Eloísa Del Castillo-Matamoros, ND, Lc, MSc, PhD ${ }^{I}$; Natalia E. Poveda, ND, \\ $\operatorname{MSc}, \operatorname{PhD}(c)^{2}$
}

n el presente número de la RCOG, se aborda una de las caras de la malnutrición, a saber, la que se da por exceso. Las autoras presentan un estudio cualitativo de etnografía enfocada para entender a profundidad las barreras que afrontan las mujeres gestantes con exceso de peso en el logro de una adecuada alimentación, en la etapa de la gestación. Este estudio concluye que hay cinco barreras entre factores proximales de la gestante (como sus hábitos alimentarios que están mediados e influenciados por factores familiares y culturales, su estado de salud actual o en gestaciones previas, y el conocimiento de las mujeres sobre alimentación y los efectos adversos del exceso de peso), factores intermedios relacionados con la atención durante el embarazo (como el rol que cumplen los prestadores de servicios de salud con relación a la educación alimentaria y nutricional), y factores de tipo estructural como la pobreza. En este sentido, lo primero que habría que decir frente a la importancia de la alimentación en la gestación es la necesidad de reconocer su complejidad frente a otros abordajes de lo nutricional, pues se trata no solo de una de las etapas de mayor vulnerabilidad alimentaria en la vida de la mujer, sino que compromete el futuro de la salud y la dieta de los niños y niñas, e incluso determina en gran medida la salud en la vida adulta (1).

1. Profesora Asociada, Observatorio de Soberanía y Seguridad Alimentaria y Nutricional, Facultad de Medicina, Universidad Nacional de Colombia, Bogotá (Colombia)

2. Programa Doctoral en Nutrición y Ciencias de la Salud, Laney Graduate School, Emory University, Atlanta (Estados Unidos).
Por ello, la situación dietética de las mujeres durante la gestación implica pensar en las condiciones de salud y nutrición en las cuales la mujer transita en su etapa pregestacional, durante la gestación, y como mujer lactante, es decir, las condiciones nutricionales que resultan de que su cuerpo responda a las altas demandas de nueve meses de gestación y dos años de lactancia materna. Pensar en la justa dimensión de lo que implica la alimentación de la mujer remitiría a acciones más integrales que preparen y protejan a la población femenina frente a este triple desafío nutricional y alimentario (1).

De hecho, el estado alimentario de un individuo y/o una población en un determinado momento de la vida es la representación de una acumulación de influencias ambientales, biológicas, sociales y comportamentales a lo largo de toda la vida. La hipótesis sobre los Orígenes Fetales de la Salud y la Enfermedad (en inglés: Developmental Origins of Health and Disease, DoHAD), propuesta por Barker en los años 80, a partir de estudios de cohortes de nacimiento en Gran Bretaña, y posteriormente apoyada por estudios en animales, pone en evidencia la importancia de los primeros días y meses de vida de un individuo en la determinación de su estado de salud y predisposición a enfermedades en el corto, mediano y largo plazo (vida adulta) (2).

La vida temprana (desde el momento de la concepción hasta alrededor del segundo año de vida) es un período sensible y de plasticidad, lo que significa que las células y órganos en formación se adaptan a los factores ambientales que afectan a la madre, por 
ejemplo, estrés, infecciones, consumo de alcohol, tabaquismo, enfermedades crónicas, obesidad, malnutrición, que a su vez determinan la susceptibilidad del individuo a cierto grado de riesgo de enfermedad en la vida adulta (3). En la gestación, el feto es especialmente susceptible a factores ambientales como la disponibilidad de nutrientes y el equilibrio energético que son imprescindibles para un crecimiento y desarrollo óptimos. Pero, no solo la gestación es una etapa de alta demanda energética y nutricional (especialmente el segundo y tercer trimestres) para la formación del feto en crecimiento, sino que es una etapa de preparación para una demanda energética aún mayor como la lactancia (4).

En términos nutricionales y metabólicos, la primera mitad de la gestación se caracteriza por ser una fase anabólica en la que el cuerpo de la mujer se adapta para mantener grandes reservas energéticas a través de ácidos grasos y proteína, y a través de cambios en la sensibilidad a la insulina, seguida en la segunda mitad de una fase catabólica en la que se da una movilización de estas reservas maternas que deben llegar al feto a través de la placenta para que este crezca adecuadamente (5). En esta primera fase, también hay una alta demanda de micronutrientes, entre los cuales destaca el ácido fólico (un elemento clave en "one-carbon metabolism”, precursor de los ácidos nucleicos requeridos para la replicación celular), el hierro (que es necesario para la formación de varias proteínas, un ejemplo es la hemoglobina, la cual es indispensable para el transporte de oxígeno y por ende la respiración celular), y el calcio (necesario para la formación ósea y comunicación intracelular) (6). Cabe mencionar que las vías bioquímicas en las que participan estos tres micronutrientes necesitan de otras vitaminas y minerales como intermediarios para que las reacciones bioquímicas se den. La mayor actividad anabólica que determina en gran medida un aumento de las demandas en los nutrientes debe ser una de las preocupaciones centrales en la atención a la gestante, en razón a la fuerte asociación con resultados adversos del desenlace de la gestación cuando la atención nutricional no es considerada prioridad o no hace parte de las acciones centrales del cuidado de la gestante (7).
Así que una alimentación y nutrición adecuadas durante el embarazo con una apropiada suplementación de los tres micronutrientes mencionados (debido a las altas demandas fisiológicas que supone la gestación humana) son necesarios para proporcionar las reservas energéticas y el pool de macro y micronutrientes que facilitan las múltiples reacciones de replicación y diferenciación celular, que en conjunto son los sustratos fisiológicos que asegurarán un crecimiento y desarrollo fetal óptimos. Una adecuada alimentación durante la etapa gestacional contribuye de manera decidida en la prevención de problemas como el retraso en el crecimiento intrauterino, el exceso de peso o el bajo peso al nacer, la morbi-mortalidad neonatal y materna durante el parto, y las complicaciones durante el mismo, que están fuertemente asociadas al estado nutricional de la mujer gestante.

Sin embargo, Colombia aún está lejos de lograr que todas las mujeres gestantes tengan un estado nutricional adecuado debido a la coexistencia de un nivel poblacional de malnutrición por déficit y por exceso. Según datos de la última Encuesta Nacional de la Situación Nutricional (ENSIN 2015), el 14,2\% de mujeres gestantes entre los 10 y 49 años tenían bajo peso, con un porcentaje de bajo peso aún mayor en gestantes adolescentes $(21,4 \%$, coeficiente de variación $\geq 20 \%$ ). La prevalencia de bajo peso solo disminuyó en un punto porcentual al comparar datos del 2010 y 2015 (8). Por su parte, el mayor porcentaje de exceso de peso se observó en gestantes adultas con una prevalencia nacional del 42,6\%, y hubo un aumento de casi 4 puntos porcentuales en el periodo 2010-2015 al comparar datos de exceso de peso en las gestantes de 10 a 49 años. En cuanto a las reservas de micronutrientes, la ENSIN 2015 evidenció las altas prevalencias de anemia (26,2\%), deficiencia de hierro (44,5\%), deficiencia de vitamina B12 (11,6\%), y deficiencia de vitamina D $(32,8 \%)$ en mujeres de 13 a 49 años en estado de gestación (8).

Esta situación del país es preocupante, ya que una amplia literatura muestra evidencia de que la malnutrición en todas sus formas se asocia con un mayor 
riesgo de morbi-mortalidad del binomio madre-hijo, un mayor riesgo de efectos adversos y complicaciones como el parto pretérmino, el bajo peso y/o la macrosomía fetal, parto por cesárea, mayor riesgo de obesidad en la infancia y de enfermedad cardiometabólica en la vida adulta, y complicaciones maternas como la preeclampsia, la diabetes gestacional, mayor retención de peso en el postparto y obesidad con un consecuente riesgo aumentado de padecer enfermedades crónicas no transmisibles en años posteriores a la gestación $(9,10)$.

Por ello, una de las tareas centrales a promover desde las directrices de política pública dirigidas a la población gestante es la vigilancia permanente de su estado nutricional, como una medida que debe proporcionar alertas tempranas que protejan a la madre y al bebé, como el centro de atención a la primera infancia, para que se les garantice la seguridad nutricional y se contribuya al logro de su derecho a la alimentación adecuada. La evaluación de la situación nutricional durante la gestación debe ser uno de los objetivos más importantes de los servicios de salud; el mantenimiento de un óptimo estado nutricional de la mujer gestante protege de manera directa el crecimiento, desarrollo y salud del futuro bebé (1).

Por otra parte, la promoción de la salud de las mujeres durante la gestación y el periodo de lactancia, así como de los niños y niñas menores de 2 años, es la pauta más importante para la prevención de la malnutrición y enfermedades crónicas no transmisibles relacionadas con la alimentación, teniendo en cuenta los contextos político, social, económico y cultural de esta población; es incluso una acción de política pública que debió ser más visible en las directrices dadas en los Objetivos de Desarrollo Sostenible y no subsumida en un objetivo global que, en muchos casos, es asumida de manera superficial por las acciones dirigidas a esta población. Orientar a las familias gestantes en la adopción de estilos de vida y prácticas de alimentación saludables, culturalmente apropiadas, que contribuyan a la prevención de la malnutrición y enfermedades crónicas no trasmisibles, en las mujeres gestantes, madres en periodo de lactancia, y niños y niñas menores de 2 años, debe ser una prioridad hoy por hoy en los programas de atención integral a la gestante para garantizar una nutrición optima de una gran proporción de la población mundial (11).

\section{REFERENCIAS}

1. Martínez García RM, Jiménez Ortega AI, Peral-Suárez A, Bermejo LM, Rodríguez-Rodríguez E. Importancia de la nutrición durante el embarazo. Impacto en la composición de la leche materna. Nutr. Hosp. 2020; 37, (N. ${ }^{\circ}$ Extra 2):38-42. https://dx.doi.org/10.20960/ nh.03355.

2. Barker DJ. The developmental origins of well-being. Philos Trans R Soc Lond B Biol Sci 2004; 359(1449):1359-66. https://doi.org/10.1098/rstb.2004.1518

3. Mandy M, Nyirenda M. Developmental Origins of Health and Disease: the relevance to developing nations. Int Health. 2018; 10(2):66-70. https://doi.org/10.1093/ inthealth/ihy006

4. King JC. Physiology of pregnancy and nutrient metabolism. Am J Clin Nutr. 2000; 71(Suppl 5):1218S-25S. https://doi.org/10.1093/ajcn/71.5.1218s

5. Tan EK, Tan EL. Alterations in physiology and anatomy during pregnancy. Best Pract Res Clin Obstet Gynaecol. 2013; 27(6):791-802. https://doi.org/10.1016/j. bpobgyn.2013.08.001

6. Mousa A, Naqash A, Lim S. Macronutrient and Micronutrient Intake during Pregnancy: An Overview of Recent Evidence. Nutrients. 2019; 11(2):443. https:// doi.org/10.3390/nu11020443

7. Observatorio de Soberanía y Seguridad Alimentaria y Nutricional. Determinantes Socioeconómicos y Alimentarios de Gestantes atendidas en la Red Pública del DC, Informe SISVAN 2016. En prensa 2016.

8. Gobierno de Colombia. Encuesta Nacional de Situación Nutricional (ENSIN) 2015. Instituto Colombiano de Bienestar Familiar, Instituto Nacional de Salud, Universidad Nacional de Colombia; 2015.

9. Kominiarek MA, Peaceman AM. Gestational weight gain. Am J Obstet Gynecol. 2017; 217(6):642-651. https:// doi.org/10.1016/j.ajog.2017.05.040 
10. Champion ML, Harper LM. Gestational Weight Gain: Update on Outcomes and Interventions. Curr Diab Rep. 2020; 20(3):11. https://doi.org/10.1007/s11892-0201296-1.

11. Instituto Colombiano de Bienestar Familiar, Organización de las Naciones Unidas para la Alimentación y la Agricultura. Manual del facilitador: Guías Alimentarias Basadas en Alimentos para Mujeres Gestantes, Madres en Periodo de Lactancia, Niños y Niñas menores de 2 años para Colombia. Bogotá: ICBF, FAO; 2018. 